The topics and relevant presentations were grouped together and dealt with in half-day sessions. Altogether, 35 lectures were held, of which 15 were delivered by invited speakers and the rest were selected high-quality contributions from the participants. The invited speakers were eminent scientists, well known in their particular fields of contemporary pharmaceutical research. The poster session consisted of 33 presentations.

Within the framework of the conference, three courses were organized for students wishing to expand their knowledge of a particular topic. The courses were run by Rolf Hilfiker (Solvias AG), Nelu Grinberg (Boehringer Ingelheim), and Stefan Dove (University of Regensburg).

The relaxing and warm atmosphere at the conference enabled a free flow of scientific information among the participants. The interaction among scientists from academic institutions and those from the industry was particularly encouraged. Valuable contacts were made and various aspects of cooperation discussed and agreed upon as a result of the conference.

The social events included a welcome reception, a social dinner, an excursion to the Krka waterfalls national park, and a visit to the famous St. Jacob's cathedral in Šibenik, which is on the UNESCO cultural heritage list.

Vladislav Tomišić <vtomisic@chem.pmf.hr> is a professor in the Faculty of Science, University of Zagreb, Croatia. Zoran Mandić <zmandic@fkit.hr>, chair of the Organizing Committee, is a professor in the Faculty of Chemical Engineering and Technology, University of Zagreb.

\section{Contemporary Chemistry for Sustainability and Economic Sufficiency}

\section{by Supa Hannongbua}

The 14th Asian Chemical Congress 2011 (14ACC), which took place in Bangkok, Thailand, 5-8 September 2011, was the largest chemical congress in Asia celebrating the International Year of Chemistry. Hosted by the Chemical Society of Thailand under the Patronage of Professor Dr. Her Royal Highness Princess Chulabhorn, the Congress was a unique and exciting forum for meeting the challenge put forth by the event's theme: "Contempary Chemistry for Sustainability and Economic Sufficiency." With 1965 attendees from 48 countries, 1262 scientific papers, 2 Nobel laureates, 23 Symposia, 5 workshops, a scientific exhibition, and many special events, the Congress was the largest chemical conference ever held in Bangkok.

The Asian Chemical Congress is the biannual congress traditionally hosted by the members of the Federation of Asian Chemical Societies (FACS), which comprises 28 chemical societies of countries and territories in the Asia Pacific. The Congress featured two Nobel laureates in chemistry: Yuan Tseh Lee (1986, Chinese Taipei) and Ada Yonath (2009, Israel) and four distinguished chemists: Yongyuth Yuthavong (Thailand), Minoru Isobe (Japan), Keiji Morokuma (Japan) and Niyazi Serdar Sariciftci (Austria), as the Plenary Lecturers.

The scientific program featured four FACS Awards Lectures:

- Foundation Lectureship Award 2011 in Inorganic Chemistry (Shie-Ming Peng, Chinese Taipei)

- FACS Distinguished Contribution to Economic Advancement Award 2011 (Pailin Chuchottaworn, Thailand)

- FACS Distinguished Contribution to Chemical Education Award 2011 (Kazuko Ogino, Japan)

- $\quad$ FACS Distinguished Young Chemist Award 2011 in Inorganic Chemistry (Wai-Yeung Wong, Hong Kong)

Following is a sampling from the 23 symposia, held in 45 concurrent sessions:

- Separation, Storage, and Utilization of $\mathrm{CO}_{2}$

- Recent Advances in Functional Materials II

- Flow Techniques and Downscaling for Analytical Sciences

- Membranes for Molecular Separation

- Medical Applications of Nano Materials

- Recent Progresses in Lab on a Chip and Its Applications

- Medicinal Chemistry

- Elsevier Symposium "General Synthetic Chemistry"

- Renewable Energy III: Artificial Photosynthesis and Hydrogen Production

- Recent Trends in Heterocyclic Compounds

- Novel Synthesis of Nano Materials and Applications 
Five workshops were held in collaboration with international organizations:

- IUPAC Workshop on Recent Advances of Natural Products (see this issue of $\mathrm{Cl}$, page 29)

- Joint Thai-UK Workshop on Frontiers in Drug Discovery Research, supported by Royal Society of Chemistry

- $\quad$ FACS workshop on Small Scale Chemistry

- UNIDO workshop on Green Industry, Organic Pollutant and Persistent Organic Pollutants

- Sandia National Laboratory workshop on Chemical Security Engagement

The scientific papers were organized in the following areas:

- Analytical and Environmental Chemistry

- Materials and Polymer Chemistry

- Physical and Theoretical Chemistry

- Organic Chemistry and Green Chemistry

- Chemical Education

- Chemistry, Nanochemistry and Catalysis

- Natural Products, Chemical Biology and Medicinal Chemistry

- Industrial Chemistry \& Innovation

A selection of the 85 papers published in the conference proceedings will be published in a special issue of Pure and Applied Chemistry on "Novelty in Green Analytical Chemistry."

The Congress offered special activities and events that celebrated IYC 2011:

- a half-day symposium on "Future Chemical Perspectives in Asia" organized by David Black featured internationally prominent chemists: Yasuhiro Uozumi (Japan), Anjali Rahatgaonkar (India), Tientong Thongpanchang (Thailand), and Zhixiang Yu (China).
- 16 IUPAC Young Chemist Awards were given out, providing partial travel support

- 2 RSC young Chemists in Analytical Chemistry were supported

- Bangkok Bank sponsored the registration fees for 100 young Thai chemists

During the 14ACC, three important meetings were held: 16th FACS General Assembly, 60th FACS Executive Committee meeting, and the 7th Asian Chemical Editorial Societies meeting. Some memorable social activities gave attendees a taste of Thai culture. The welcome reception and banquet, organized by Rajamangala University of Technology Thanyaburi, included traditional dance, music, and delicious food.

The 14ACC was made possible by the financial support and collaboration of the Thailand Convention and Exhibition Bureau, the Federation of Asian Chemical Societies, IUPAC, The Elsevier Properties SA, Kavli Foundation, and Royal Society of Chemistry. The conference was also supported by the departments of chemistry of seven local universities, Chulalongkorn University, Kasetsart University, Chiang Mai University, Khon Kaen University, Mahidol University, Prince of Songkla University and Rajamangala University of Technology Thanyaburi. In addition, a number of private companies, including IRPC Public Company Limited, Bangkok Bank Public Company Limited, the Siam Cement Group, Mettler-Toledo (Thailand) Limited, L'OREAL (Thailand) Limited, and others made important contributions to the Congress.

Supa Hannongbua <fscisph@ku.ac.th>, chair of the program committee, is head of the Chemistry Department at Kasetsart University in Bangkok, Thailand.

\section{www.14acc.org}

